\title{
LOWER ESTIMATE IN LITTLEWOOD'S CONJECTURE ON THE MEAN SPHERICAL DERIVATIVE OF A POLYNOMIAL AND ITERATION THEORY
}

\author{
A. E. EREMENKO
}

(Communicated by Clifford J. Earle, Jr.)

\begin{abstract}
Lewis and Wu have proved J. E. Littlewood's conjecture of the growth of the spherical derivative of polynomials. We apply the theory of complex iteration to show that this conjecture is sharp in a qualitative sense.
\end{abstract}

Let $f$ be a complex polynomial. Denoted by $\rho(f)=\mid f^{\prime} / 1\left(1+\left|f^{2}\right|\right)$ its spherical derivative, let $D$ be the unit disc, and set

$$
\Phi(f)=\iint_{D} \rho(f(z)) d x d y .
$$

Littlewood [L] remarked that $\Phi(f) \leq \pi \sqrt{n}$ for all polynomials of degree $n$. He asked for good upper and lower bounds for the quantity $\phi(n)=$ $\sup \{\Phi(f): \operatorname{deg} f=n\}$ and conjectured that

$$
\phi(n) \leq c n^{\alpha}
$$

for some $\alpha<\frac{1}{2}$. It was proved in [ES1] that $\phi(n)=o(\sqrt{n}), n \rightarrow \infty$ and later J. Lewis and J. M. Wu [LeW] obtained the estimate (1) with $\alpha=2^{-1}-2^{-264}$ by improving the potential-theoretic method of [ES1, ES2]. Upper bounds for $\phi(n)$ have very interesting consequences in value distribution theory of entire functions [L, ES1, ES2].

The lower estimate in [L] was $\phi(n) \geq c(\delta)(\log n)^{1 / 2-\delta}$ for every $\delta>0$. Then W. Hayman $[\mathrm{H}]$ improved this to $\phi(n) \geq c \log n$.

We shall prove in this note that $\phi(n) \geq c n^{\kappa}$ for some $\kappa>0$. To this end we use complex iteration theory (for a brief account of this theory see for example [B, EL]). Denote by $f^{n}$ the $n$th iterate of a polynomial $f$. The Julia set $J(f)$ is the closure of repelling periodic points of $f$. A polynomial $f$ is called the hyperbolic if $f^{\prime}(z) \neq 0, z \in J(f)$. In this case the closure of the orbit of critical points of $f$ does not intersect $J(f)$. For example $f(z)=z^{2}+\lambda$ is

Received by the editors December 1, 1989 and, in revised form, June 30, 1990.

1980 Mathematics Subject Classification (1985 Revision). Primary 30C10; Secondary 30B20, $30 \mathrm{D} 15$. 
hyperbolic if $\lambda$ is in the interior of the cardioid:

$$
C=\left\{\frac{e^{i \theta}}{2}-\frac{e^{2 i \theta}}{4}: 0<\theta \leq 2 \pi\right\} .
$$

The orbit of the unique critical point of such a polynomial tends to the attracting fixed point.

Let $f$ be a hyperbolic polynomial. Consider a covering of $J=J(f)$ by closed squares $\mathscr{U}_{j}$ of diameter $r>0$ centered at the points $z_{j} \in J$ and satisfying int $\mathscr{U}_{j} \cap$ int $\mathscr{U}_{i}=\varnothing, j \neq i$. Denote by $\mathscr{U}_{j}^{1}$ the squares with the centers $z_{j}$ having diameter $2 r$ and sides parallel to those of $\mathscr{U}_{j}$. Choose the diameter $r$ so small that $\mathscr{U}^{1}=\bigcup_{j} \mathscr{U}_{j}^{1}$ contains no critical values of iterates. This is possible because critical values of $f^{n}$ are contained in the orbit of critical points of $f$ and $f$ is hyperbolic. All branches of $f^{-n}$ are single valued and univalent in $\mathscr{U}_{j}^{1}$. They map $\mathscr{U}_{j}$ onto some regions $V_{k}$ which cover $J$ and int $V_{k} \cap$ int $V_{l}=\varnothing, k \neq l$. Choose $a_{k}$ in $V^{k}$ such that $f^{n}\left(a_{k}\right)=z_{j}$ for some $j$. On applying the Koebe distortion theorem to each branch of $f^{-n}$ in $\mathscr{U}_{j}^{1}$, we obtain

$$
\text { area } V_{k} \asymp\left|\left(f^{n}\right)^{\prime}\left(a_{k}\right)\right|^{-2}
$$

and

$$
\left|\left(f^{n}\right)^{\prime}(z)\right| \asymp\left|\left(f^{n}\right)^{\prime}\left(a_{k}\right)\right|, \quad z \in V_{k},
$$

$\left(x \asymp y\right.$ means $c_{1} x \leq y \leq c_{2} x$ with constants $c_{1}$ and $c_{2}$ depending only on $\left.\left\{\mathscr{U}_{j}\right\}\right)$. If $z \in V=\bigcup_{k} V_{k}$ then $f^{n}(z) \in \mathscr{U}^{1}$ and thus $f^{n}$ is bounded on $V$ uniformally in $n$. Futhermore we can suppose that $J \subset \mathscr{D}=\{z:|z|<1\}$ and consequently $V \subset \mathscr{D}$ for large $n$. Using (2) and (3) we obtain

$$
\Phi\left(f^{n}\right) \geq c \iint_{V}\left|\left(f^{n}\right)^{\prime}\right|=c \sum_{k} \iint_{V_{k}}\left|\left(f^{n}\right)^{\prime}\right| \asymp \sum_{j} \sum_{f^{n}(z)=z_{j}}\left|\left(f^{n}\right)^{\prime}(z)\right|^{-1} .
$$

It is known that for every hyperbolic polynomial $f$ and every $t \in \mathbb{R}$, the following limit exists:

$$
P(t)=P_{f}(t)=\lim _{n \rightarrow \infty} \frac{1}{n} \log \sum_{f^{n}(z)=a}\left|\left(f^{n}\right)^{\prime}(z)\right|^{-t} .
$$

This limit is called pressure and it does not depend on $a$. (See $[\mathrm{Bo}, \mathrm{R}]$ ). It follows from (4) that

$$
\Phi\left(f^{n}\right) \geq c \exp \left(n P_{f}(1)\right)=c\left(\operatorname{deg} f^{n}\right)^{\kappa},
$$

where $\kappa=P_{f}(1) / \log \operatorname{deg} f$. D. Ruelle [R] proved that $t \mapsto P_{f}(t)$ is a decreasing convex function. Moreover $P_{f}(\delta)=0$, where $\delta$ is the Hausdorff dimension of $J$. The derivative $d P_{f} / d t$ is negative at $t=\delta$. We conclude that $P_{f}(1)>0$ if $\delta>1$. This is the case for example if $f(z)=z^{2}+\lambda$ and $\lambda$ is in the interior of the above-mentioned cardioid $C$. 
Note that a slightly more accurate computation shows that $\Phi\left(f^{n}\right) \asymp\left(\operatorname{deg} f^{n}\right)^{\kappa}$ where $\kappa=P_{f}(1) / \log \operatorname{deg} f$.

Two natural questions arise:

(1) What is $\sup \left\{P_{f}(1): f(z)=z^{2}+\lambda\right\}$ ?

(2) Define the Littlewood order of a sequence of polynomials $P_{n}$ by

$$
\varlimsup_{n \rightarrow \infty} \log \Phi\left(P_{n}\right) / \log \operatorname{deg} P_{n} \text {. }
$$

Is it true that the largest possible order occurs if $P_{n}$ are iterates of a polynomial $f$ ?

\section{REFERENCES}

[B] P. Blanchard, Complex analytic dynamics on the Riemann sphere, Bull. Amer. Math. Soc. 11 (1984), 85-141.

[Bo] R. Bowen, Equilbrium states and the ergodic theory of Anosov diffeomorphisms, Lecture Notes in Math., vol. 470, Springer-Verlag, Berlin and New York, 1975.

[EL] A. Eremenko and M. Lyubich, Dynamics of analytical transformations, Algebra and Analysis 1 (1989), 1-70 (Russian); English transl.: Leningrad Math. J. 1 (1990), 563-634.

[ES1] A. Eremenko and M. Sodin, On a conjecture of Littlewood and value distribution of entire functions, Funct. Anal. Appl. 20 (1986), 71-72.

[ES2] _ Proof of a conditional theorem of Littlewood on the distribution of values of entire functions, Math. USSR Izvestiya 30 (1988), 395-402.

[H] W. Hayman, On a conjecture of Littlewood, J. Anal. Math. 36 (1979), 75-95.

[L] J. E. Littlewood, On some conjectural inequalities with applications to the theory of intergral functions, J. London Math. Soc. 27 (1952), 387-393.

[LeW] J. L. Lewis and Jang-Mei Wu, On conjectures of Arakelyan and Littlewood, J. Anal. Math. 50 (1988), 259-283.

[R] D. Ruelle, Repellers for real analytic maps, Ergodic Theory Dynamical Systems 2 (1982), 99-107.

Department of Mathematics, Purdue University, West Lafayette, Indiana 47907 